\title{
Estadios y procesos de cambio en drogodependientes en tratamiento ambulatorio
}

\author{
SANTOS DIEZ, P.(1); GONZÁLEZ MARTÍNEZ, G. ${ }^{(2) ; ~ F O N S ~ B R I N E S, ~ M . R . ~}{ }^{(2)}$; \\ FORCADA CHAPA, R. ${ }^{(3)}$; ZAMORANO GARCÍA, C. ${ }^{(4)}$
}

\author{
Unidad de Conductas Adictivas, Área de Salud n 13. Consellería de Sanidad. Generalitat Valenciana. \\ (1) Doctor en Psicología. U.C.A. Xàtiva. Conselleria de Sanitat. Generalitat Valenciana. (2) Psicólogo/a. Investigador-colaborador U.C.A. Xàtiva. \\ (3) Médico-Responsable U.C.A. Xàtiva. (4) Enfermera U.C.A. Xàtiva.
}

Enviar correspondencia a:

Patxi Santos Diez, U.C.A. Xàtiva. Hospital Antiguo. 46800. Xàtiva. Valencia. Correo Electrónico: patxisan@correo.cop.es

\section{RESUMEN}

Con la finalidad de describir los estadios y procesos de cambio que presentan los sujetos drogodependientes que demandan tratamiento en una Unidad de Conductas Adictivas (U.C.A.) del Área de Salud $n^{\circ} 13$ de Xàtiva (Valencia), se estudió una muestra de 106 varones y 10 mujeres adictos a diferentes sustancias. Al inicio de tratamiento, el psicólogo de la U.C.A. evaluó el estadio de motivación al cambio, mientras que los procesos de cambio fueron evaluados mediante el Inventario de Procesos de Cambio (I.P.C.) de A.Tejero y J. Trujols, 1993.

Los resultados muestran la predominancia de los sujetos preparados para la acción (51.7 \%), aunque un alto porcentaje de sujetos $(30.1 \%)$ se encuentran en precontemplación o contemplación. Respecto a los procesos de cambio, la mayor puntuación la obtiene la reevaluación ambiental. Excepto en dos procesos de cambio (liberación social y control de estímulos), la puntuación obtenida es superior a la media. De los resultados se desprende que la intervención psicológica debería dirigirse hacia el aumento de la atribución interna y capacidad de decisión sobre su conducta adictiva, así como hacia la reestructuración del ambiente.

Palabras clave: Modelo transteórico, estadios de cambio, procesos de cambio, drogodependencias.

\section{SUMARY}

A study was carried out to describe the stages and processes of change presented by drug addicts who request treatment in the Addictive Behaviours Unit (ABU) of Health Area No. 13 in Xàtiva (Valencia). The study was carried out with a sample of 106 men and 10 women addicted to several substances. At the beginning of the treatment, the $A B U$ psychologist assessed the state of motivation for the change, whereas the processes of change were evaluated by the Processes of Change Inventory (P.C.I.) by A. Tejero and J. Trujols (1993).

The results show the predominance of the individuals prepared for the action $(51.7 \%)$, although a high percentage $(30.1 \%)$ were in a preconsideration or consideration phase. Regarding the processes of change, environmental reassessment obtained the highest score. Apart from two processes of change (social liberation and control of stimuli), the score obtained was higher than the average. What can be inferred from these results is that psychological intervention must be focused on increasing internal attribution and the ability to decide on their addictive behavior, as well as on the restructuration of the environment.

Key words: Transtheoretical model, stages of change, processes of change, drug-dependences.

\section{INTRODUCCIÓN.}

$\mathbf{E}$ n los últimos años estamos asistiendo y participando en un gran discurso en torno al modelo transteórico de Prochaska y DiClemente' y su aplicación a la clínica de las conductas adictivas. Un gran número de profesionales adopta este modelo como marco explicativo del cambio intencional de una conducta adictiva; y basándose en él se han desarrollado diferentes estrategias y elementos terapéuticos que ayudan al sujeto a progresar hacia estadios de cambio más avanzados, como por ejemplo la entrevista motivacional².

El modelo de Prochaska y DiClemente es de tipo tridimensional, y proporciona una concepción global y al mismo tiempo diferenciada del cambio en el campo de las conductas adictivas, integrando lo que sus autores denominan estadios, procesos y niveles de cambio. Los cinco estadios de cambio representan la estructura o dimensión temporal o evolutiva del modelo transteórico: precontemplación, contemplación, preparación, actuación y mantenimiento. 
Prochaska y DiClemente identifican diez procesos básicos de cambio que posibilitan comprender cómo sucede el cambio intencional, es decir, el cambio de intenciones, actitudes y conductas:

1.- Aumento de la concienciación. Consiste en un incremento del procesamiento de información sobre la problemática asociada al consumo y a los beneficios de modificarla.

2.- Autorreevaluación. Es la valoración afectiva y cognitiva del impacto de la conducta adictiva sobre los valores y manera de ser de uno mismo, así como el reconocimiento de la mejoría significativa que representaría para su vida el abandono de la conducta adictiva.

3.- Reevaluación ambiental. Consiste en una valoración del estado actual de las relaciones interpersonales y en un reconocimiento de las consecuencias positivas que produciría el cese de la conducta adictiva respecto a dichas relaciones interpersonales, familiares y de amistad.

4.- Relieve dramático. Es la experimentación y expresión de reacciones emocionales elicitadas por la observación y/o advertencias respecto a los aspectos negativos asociados a la conducta adictiva.

5.- Autoliberación. Representa un compromiso personal; un aumento de la capacidad del sujeto para decidir y elegir. Requiere la creencia de que uno puede ser un elemento esencial en el proceso de cambio de la conducta adictiva, ya que se poseen o se pueden desarrollar las habilidades necesarias para efectuar un cambio exitoso.

6.- Liberación social. Representa un aumento en la capacidad del sujeto para decidir y escoger, propiciado por una toma de conciencia tanto de la representación social de la conducta adictiva como de la voluntad social de combatirla mediante el incremento del repertorio personal de alternativas adaptativas.

7.- Manejo de contingencias. Es una estrategia conductual que incrementa la probabilidad de que determinada conducta relativa al cambio ocurra. Consiste en el auto y/o hetero-refuerzo de dicha conducta.

8.- Relaciones de ayuda. Representa la existencia y utilización del apoyo social (familia, amistades, etc.) que pueda facilitar el proceso de cambio de la conducta adictiva.

9.- Contracondicionamiento. Estrategia cognitivo-conductual cuyo objetivo es modificar la respuesta (cognitiva, fisiológica y/o motora) elicitada por estímulos condicionados a la conducta adictiva u otro tipo de situaciones de riesgo, generando y desarrollando para ello conductas alternativas.

10.- Control de estímulos. Consiste básicamente en evitar la exposición a situaciones de alto riesgo para el consumo.

Los procesos de cambio consisten en actividades encubiertas o manifiestas iniciadas o experimentadas por un individuo para modificar su hábito adictivo.
Por último, los niveles de cambio se presentan como una organización jerárquica en la que se interrelacionan los procesos psicológicos susceptibles de ser tratados. Es decir, se refieren a qué es necesario cambiar para abandonar la conducta adictiva. Estos niveles son: síntoma/situación, cogniciones desadaptativas, conflictos actuales interpersonales, conflictos de familia/sitemas y conflictos intrapersonales.

El modelo transteórico de Prochaska y DiClemente es uno de los que mayor soporte empírico ha recibido en relación con su capacidad descriptiva, explicativa y predictiva del proceso de cambio en el ámbito de las conductas adictivas $3,4,5,6,7,7,9,10,11,12$ (por citar sólo algunos).

La revisión documental, si bien ofrece algunas investigaciones sobre estadios de cambio en población española, permite localizar muy pocas centradas en el estudio de los procesos de cambio.

En relación a los estadios de cambio, algunos estudios realizados con adictos a opiáceos señalan que entre el $60-70 \%$ de los sujetos que acuden en demanda de tratamiento se ubican en el estadio de contemplación ${ }^{12,13}$, encontrándose resultados similares en adictos a cocaína ${ }^{14}$. Otros trabajos llevados a cabo con alcohólicos ${ }^{15,16}$ señalan porcentajes alrededor del $50 \%$ de los sujetos en dicho estadio. En fumadores se obtienen resultados entre el $30 \%$ y el $40 \%$ en el estadio de contemplación ${ }^{17}$. Finalmente, alrededor del $60 \%$ de los jugadores patológicos que demandan tratamiento en diversas Unidades de Conductas Adictivas de la Comunidad Valenciana se ubican en el estadio de contemplación ${ }^{18}$.

\section{OBJETIVOS:}

1.- Identificar los estadios de cambio en los que se ubican los sujetos

que demandan tratamiento.

2.- Identificar los procesos de cambio que presentan los sujetos al

inicio de tratamiento.

\section{MUESTRA}

La muestra objeto del presente estudio está compuesta por 116 sujetos (10 mujeres y 106 varones) que demandaron tratamiento por primera vez en la Unidad de Conductas Adictivas del área de salud $n^{\circ} 13$ de Xàtiva durante el periodo comprendido entre julio y noviembre de 1999 y que cumplían criterios diagnósticos de dependencia de sustancias (DSM-IV).

Se excluyeron los sujetos que presentaban comorbilidad psiquiátrica grave. 


\section{MATERIAL Y MÉTODO}

El estadio de motivación al cambio fue evaluado por el psicólogo de la U.C.A. al inicio de tratamiento. Para estudiar el estadio de cambio se intercalaron durante la primera entrevista las siguientes preguntas: ¿consumes en la actualidad?, ¿estás considerando seriamente dejar de consumir en los próximos 6 meses?, ¿te planteas dejar de consumir en los próximos 30 días?, ¿has dejado de consumir, al menos un día, durante el año pasado? y ¿cuánto tiempo llevas sin consumir?. Se trata una adaptación de un sistema de clasificación categorial basado en una serie de preguntas mutuamente excluyentes desarrollado por DiClemente et al. ${ }^{19}$ y Prochaska y DiClemente ${ }^{20}$ para fumadores. El precontemplador contestaría que está consumiendo en la actualidad y no considera seriamente dejar de consumir en los próximos 6 meses. El contemplador consume en la actualidad pero consideran seriamente dejar de consumir en los próximos 6 meses. El preparado para el cambio consume en la actualidad pero está planeando dejar de consumir en los próximos 30 días y ha estado abstinente por un período de al menos un día durante el año anterior; aquellos que tienen la intención de dejar de consumir pero que no reúnen todos los criterios citados son considerados contempladores. Los sujetos ubicados en acción son aquellos que no consumen en la actualidad y presentan una abstinencia inferior a seis meses. Por último, los sujetos que presenta una abstinencia superior a 6 meses se ubicarían en mantenimiento.

Los procesos de cambio fueron evaluados mediante la administración del Inventario de Procesos de Cambio (I.P.C. ${ }^{10}$. Este inventario es un autoinforme que consta de 40 ítems, cuatro para cada una de las diez escalas correspondientes a los diez procesos de cambio, desarrollado conforme a las directrices que se desprenden del trabajo de Prochaska y DiClemente. Se responde mediante una escala tipo Likert de 4 puntos que mide la frecuencia con que utilizan las estrategias cognitivo-conductuales. La puntuación máxima en cada proceso es doce. Su estructura factorial, consistencia interna y eficacia discriminativa están evaluadas en población drogodependiente española y son satisfactorias ${ }^{21}$. Su fiabilidad (consistencia interna) es de 0,87.

Las variables sociodemográficas, toxicológicas, sanitarias, etc. fueron registradas al inicio del tratamiento en una plantilla elaborada previamente.

El análisis y tratamiento de todos los datos han sido realizados mediante el paquete estadístico SPSS.

\section{RESULTADOS}

El perfil de la muestra vendría caracterizado por un varón de 31 años, soltero, que vive con la familia de origen; con un nivel de estudios primarios, de profesión obrero no cualificado, en situación laboral de activo; sin problemas judiciales; que acude al centro remitido por Atención Primaria, Unidades de Salud Mental o iniciativa propia; acude solo, acompañado por su madre o pareja.

El relación a las variables toxicológicas medidas, es importante señalar cómo la demanda de sujetos que presentan dependencia de alcohol es superior al de otras conductas adictivas. Más de la mitad de la muestra no han recibido tratamiento previo, y cerca del $50 \%$ no presentan abstinencia previa. El $50.9 \%$ de la muestra presenta antecedentes personales de otras conductas adictivas, predominando entre ellos los de cocaína (19.8\%). Por otro lado, prácticamente un tercio de los sujetos presenta antecedentes familiares de dependencia.

Aunque predomina la ausencia de antecedentes personales de psicopatología, es importante resaltar cómo más del $30 \%$ de la muestra ha padecido algún trastorno psicopatológico con anterioridad al inicio de tratamiento; el $12.1 \%$ presenta antecedentes familiares de psicopatología.

Con relación a los resultados obtenidos en la evaluación del estadio de cambio en el que se ubican los sujetos al inicio de tratamiento, señalar que predominan los sujetos preparados para la acción, es decir, el adicto toma la decisión y se compromete a abandonar su conducta adictiva y realiza algunos pequeños cambios conductuales (disminuir la dosis consumida, por ejemplo), aunque no cumpla criterios necesarios para considerar que se halle en actuación (abstinencia total, por ejemplo). Un alto porcentaje de sujetos (30.1\%) se encuentran en precontemplación o contemplación, es decir, no se plantean modificar su conducta adictiva ya que no son conscientes de que dicha conducta represente un problema; o son conscientes pero no han desarrollado todavía un compromiso firme de cambio. Ver gráfica 1.

Respecto a los procesos de cambio, indicar que la puntuación mayor la obtiene la reevaluación ambiental, lo cual indica que existe una alta valoración del impacto de la conducta adictiva en sus relaciones interpersonales y el reconocimiento de consecuencias positivas en las mismas derivadas de la abstinencia. Es importante señalar cómo excepto en dos procesos de cambio (liberación social y control de estímulos), la puntuación obtenida en el resto es superior a la media (mayor de 6), significando que no existe una toma de conciencia sobre su propia capacidad para decidir y escoger (liberación social), ni reestructuración del ambiente para disminuir la probabilidad de exponerse 
TABLA I: CARACTERÍSTICAS TOXICOLÓGICAS

\begin{tabular}{|c|c|c|c|}
\hline \multicolumn{2}{|c|}{ VARIABLES } & $\mathbf{N}$ & $\%$ \\
\hline SUSTANCIA PRINCIPAL & $\begin{array}{l}\text { Heroína } \\
\text { Alcohol } \\
\text { Cocaína } \\
\text { Cannabis } \\
\text { Otras }\end{array}$ & $\begin{array}{r}35 \\
42 \\
29 \\
8 \\
2\end{array}$ & $\begin{array}{r}30.2 \\
36.2 \\
25.0 \\
6.9 \\
1.7\end{array}$ \\
\hline $\begin{array}{l}\text { CONSUMO CONCOMITANTE } \\
\text { DE OTRAS SUSTANCIAS }\end{array}$ & $\begin{array}{l}\text { Ninguna } \\
\text { Alcohol } \\
\text { Cocaína } \\
\text { Otras }\end{array}$ & $\begin{array}{l}41 \\
31 \\
30 \\
14\end{array}$ & $\begin{array}{l}35.3 \\
26.7 \\
25.9 \\
12.1 \\
\end{array}$ \\
\hline TRATAMIENTOS PREVIOS & $\begin{array}{l}0 \\
1 \\
2 \\
3 \text { ó más. }\end{array}$ & $\begin{array}{r}63 \\
29 \\
15 \\
9\end{array}$ & $\begin{array}{r}54.3 \\
25.0 \\
12.9 \\
7.8 \\
\end{array}$ \\
\hline $\begin{array}{l}\text { RECURSO/STRATAMIENTO/S } \\
\text { ANTERIOR/ES }\end{array}$ & $\begin{array}{l}\text { Sin tratamiento previo } \\
\text { Unidad de Conductas } \\
\text { Adictivas } \\
\text { Comunidad Terapéutica } \\
\text { Unidad de Salud Mental } \\
\text { Centros no oficiales }\end{array}$ & $\begin{array}{r}63 \\
23 \\
6 \\
5 \\
19\end{array}$ & $\begin{array}{r}54.3 \\
19.8 \\
5.2 \\
4.3 \\
16.4\end{array}$ \\
\hline $\begin{array}{l}\text { TIEMPO MÁXIMO } \\
\text { DE ABSTINENCIA PREVIA }\end{array}$ & $\begin{array}{l}0 \\
1-3 \text { meses } \\
4-6 " \\
7-9 " \\
10-12 " \\
13 \text { o más " }\end{array}$ & $\begin{array}{r}53 \\
28 \\
4 \\
9 \\
7 \\
15\end{array}$ & $\begin{array}{r}45.7 \\
24.1 \\
3.4 \\
7.8 \\
6.0 \\
12.9\end{array}$ \\
\hline $\begin{array}{l}\text { ANTECEDENTES PERSONALES DE } \\
\text { OTRAS CONDUCTAS ADICTIVAS }\end{array}$ & $\begin{array}{l}\text { No } \\
\text { Alcohol } \\
\text { Cocaína } \\
\text { Heroína } \\
\text { Otras }\end{array}$ & $\begin{array}{r}57 \\
14 \\
23 \\
2 \\
20\end{array}$ & \begin{tabular}{r|}
49.1 \\
12.1 \\
19.8 \\
1.7 \\
17.2
\end{tabular} \\
\hline $\begin{array}{l}\text { ANTECEDENTES FAMILIARES DE } \\
\text { ADICCIÓN }\end{array}$ & $\begin{array}{l}\text { Ninguna } \\
\text { Alcohol } \\
\text { Juego } \\
\text { Drogas ilegales }\end{array}$ & $\begin{array}{r}88 \\
13 \\
3 \\
12\end{array}$ & $\begin{array}{r}75.9 \\
11.2 \\
2.6 \\
10.3\end{array}$ \\
\hline
\end{tabular}

\section{GRAFICO 1}

mantenimiento

$4,3 \%$

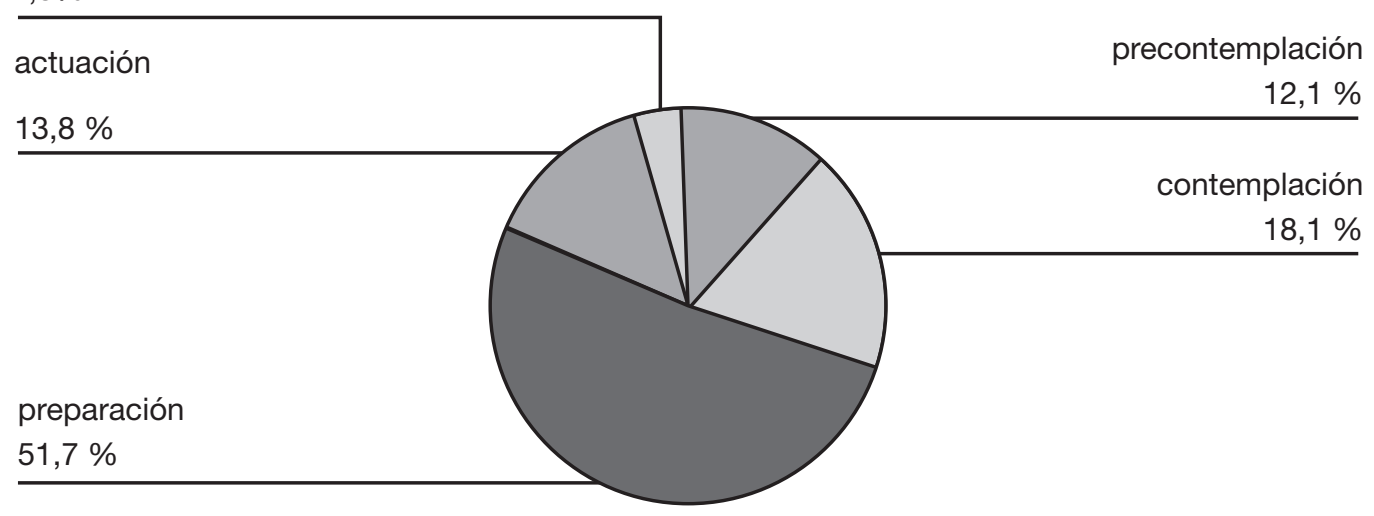


TABLA II: PROCESOS DE CAMBIO

\begin{tabular}{|l|c|c|}
\hline & Media & Desviación Típica \\
\hline Aumento de la concienciación & 6.170 & 3.140 \\
Autoliberación & 8.525 & 3.038 \\
Liberación Social & 5.737 & 3.076 \\
Autorrevaluación & 7.848 & 3.173 \\
Reevaluación Ambiental & 9.263 & 2.798 \\
Contracondicionamiento & 6.101 & 3.460 \\
Control de Estímulos & 4.808 & 3.585 \\
Manejo de Contingencias & 7.758 & 3.176 \\
Relieve Dramático & 6.667 & 3.080 \\
Relaciones de Ayuda & 7.192 & 2.834 \\
\hline Puntuación máxima: 12. & & \\
\hline
\end{tabular}

a situaciones de alto riesgo para consumir (control de estímulos). Ver tabla II.

\section{CONCLUSIONES}

Los resultados sociodemográficos coinciden en términos generales con los descritos por otros autores $^{12,13}$. Cabría destacar la elevada proporción de sujetos que refiere no tener problemas judiciales, teniendo en cuenta que un $54.3 \%$ de la muestra presenta adicción a sustancias ilegales.

En cuanto a las características toxicológicas, nos llama la atención la gran proporción de sujetos que no han realizado tratamientos previos $(54.3 \%)$; lo cual podría venir determinado por tratarse de una U.C.A. de reciente creación en un área en el que no existía dicho recurso. Por otro lado, observamos cómo los sujetos de nuestro estudio consumen menos sustancias concomitantemente a la principal que los de otros trabajos ${ }^{14,22}$.

Nos parece novedoso que casi la mitad de los sujetos que componen la muestra han sido derivados desde Unidades de Salud Mental o Atención Primaria (recursos sanitarios públicos). Consideramos que ello es debido, en gran parte, a la inclusión en la Comunidad Valenciana de las Unidades de Conductas Adictivas dentro de la red de Atención Primaria.

El hecho de que el $51.7 \%$ de la muestra se ubique en el estadio de preparación al inicio de tratamiento refleja que gran parte de los sujetos está altamente motivada hacia el cambio. Estos resultados difieren de los encontrados por otros autores $12,13,14,15,16,18$, quienes encuentran una proporción de sujetos menor en el estadio de preparación y actuación, y mayor en los estadios de precontemplación y contemplación. Creemos que ello puede ser debido a diferentes factores: diferentes tamaños de las muestras, diferentes varia- bles sociodemográficas y procedencia de los sujetos, al hecho de que la mitad de la muestra viene derivada desde atención primaria o salud mental lo cual podría influir en la motivación del sujeto, etc. Consideramos importante continuar esta línea de investigación con la finalidad de perfilar mejor estos resultados.

A nivel clínico consideramos que el conocimiento de los procesos de cambio que presenta el sujeto al inicio de tratamiento, puede servir para mejorar la intervención en conductas adictivas. De los resultados obtenidos en los procesos de cambio se desprende que la intervención psicológica debería ir dirigida, en el ámbito general, hacia el aumento de la atribución interna y capacidad para decidir sobre su conducta adictiva y conductas alternativas (liberación sociah, así como hacia la reestructuración del ambiente para que la probabilidad de que un determinado estímulo condicionado a la conducta adictiva se reduzca significativamente (control de estímulos). Evidentemente, se deberán estudiar en el ámbito individual los procesos de cambio que está utilizando el sujeto para potenciar (a partir del modelo de Prochaska y DiClemente) aquellos que sean más adecuados para su evolución hacia el estadio de actuación.

Consideramos adecuado, en futuras investigaciones, estudiar la existencia de diferencias en los estadios y procesos de cambio en función de la sustancia. Es decir, si existen determinados estadios y procesos de cambio específicos para cada una de las adicciones a sustancias.

Aunque los estadios de cambio ya han sido estudiados por otros autores con anterioridad, no ocurre lo mismo con los procesos de cambio, por lo que la principal aportación del presente estudio radica en ofrecer información relativa sobre los mismos en población drogodependiente española dada la escasa bibliografía que existe al respecto, así como la aportación de información de valor heurístico para futuras investigaciones. 


\section{BIBLIOGRAFÍA}

Prochaska, J.O. y DiClemente, C.C. (1982): Transtheoretical therapy: Toward a more integrative model of change. Psychotherapy: Theory, Research, and Practice, 19, 276-288.

Miller, W.R. y Rollnick, S. (1999): La entrevista motivacional. Ed. Paidós.

Brownell, K.D., Marlatt, G.A., Lichtenstein, E. y Wilson, G.T. (1986): Understanding and preventing relapse. American Psychologist, 41, 765-782.

Raw, M. (1986): Smoking cessation strategies. En W.R. Miller y N. Heather (Eds.), Treating addictive behaviors: Processes of change (pp.279-287). New York: Plenum Press.

Marlatt, G.A., Baer, J.S., Donovan, D.M. y Kivlahan, D.R. (1988): Addictive behaviors: Etiology and treatment. Annual Review of Psychology, 39, 223-252.

Baer, J.S. y Lichtenstein, E. (1988): Cognitive assessment in smoking cessation. En D.M. Donovan y G.A. Marlatt (Eds.), Assessment of addictive behaviors. New York, Guildford Press.

Prochaska, J.O., DiClemente, C.C. y Norcross, J.C. (1992): In search of how people change. Applications to addictive behaviors. American Psychologist, 47, 1102-1114.

Scheneider, R.J. y Khantzian, E. (1992): Psychotherapy and patient needs in the treatment of alcohol and cocaine abuse. En M. Galanter (Ed.), Recent developments in alcoholism (vol. 10, pp. 165-178). New York: Plenum Press

Shafer, H.J. (1992): The psuchology of stage change: the transition from addiction to recovery. En J.H. Lowinson, P. Ruiz, R.B. Millman y J.G. Langrod (Eds.), Substance abuse: A comprhensive textbook ( $2^{\mathrm{a}}$ ed.), Baltimore, Williams and Wilkins.

Tejero, A. y Trujols, J. (1994): El modelo transteórico de Prochaska y DiClemente: Un modelo dinámico de cambio en el ámbito de las conductas adictivas. En J.L. Graña (Ed.), Conductas adictivas. Teoría, evaluación y tratamiento (pp. 1-43). Madrid: Debate.

Prochaska, J.O. (1995): Common problems: Common solutions. Clinical Psychology: Science and Practice, 2, 101-105.

Albiach, C.; Llinares, M.C.; Palau, C. y Santos, P. (2000): Adherencia en heroinómanos: la potencia predictiva de los estadios de cambio evaluados durante la admisión al tratamiento. Adicciones, vol. 12, n 2, pags. 225234.

Albiach, C.; Llinares, M.C.; Santos, P. y Palau, C. (1996): Estudio descriptivo sobre sujetos heroinómanos que demandan asistencia en servicios ambulatorios: eva- luación de los estadios de cambio. En II Jornadas sobre drogodependencias. Ed.: Universidad Politécnica de Valencia.

Santos, P.; Llinares, C; Palau, C.; Albiach, C. (1998): Adherencia al tratamiento en una muestra de adictos a cocaína: Posibles variables predictoras. Libro de Comuninaciones del V Encuentro Nacional sobre drogodependencias y su enfoque comunitario. Pag. 171 - 172. Ed. Centro Provincial de Drogodependencias de Cádiz; D.P.C.; J.A.; P.N.D. y F.A.D.

Llinares, M.C.; Palau, C.; Albiach, C. y Santos, P. (1996): Alcohólicos que demandan tratamiento en centros ambulatorios: la motivación al cambio. En Il Jornadas sobre drogodependencias. Ed.: Universidad Politécnica de Valencia.

Llinares, C.; Santos, P.; Albiach, C; Palau, C. (1999): La adherencia al tratamiento en una muestra de 172 alcohólicos: seguimiento durante un año y variables predictoras. Libro de Comunicaciones del VI Encuentro Nacional sobre drogodependencias y su enfoque comunitario. Pag. 193 - 206. Ed. C.P.D. de Cádiz; D.P.C.; J.A.; P.N.D.; F.A.D. y F.A.A.D.

Prochaska, J.O.; Velicer, W.F.; DiClemente, C.; Guadagnoli, E y Rossi, J.S. (1991): Patterns of change: Dynamic Typology applied to the smoking cessation. Multivariate Behavioral Research, 26, 83-107.

Camacho, I.; Llinares, M.C.; Santos, P. y Palau, C. (1999): Perfil psicosocial del jugador patológico que demanda tratamiento en UCAs de la Comunidad Valenciana: estudio muticéntrico. Poster VII Congreso Nacional de la Sopciedad Española de Toxicomanías. Resumen en la revista Trastornos Adictivos. Vol 1, n 3-4.

DiClemente, C.C., Prochaska, J.O., Fairhurst, S.K., Velicer, W.F., Velasquez, M.M. y Rossi, J.S. (1991): The process of smoking cessation: An analysis of precontemplation, contemplation, and preparation stages of change, Journal of Consulting and Clinical Psychology, 59, 295-304.

Prochaska, J.O. y DiClemente, C.C. (1992): Stages of change in the modification of problem behaviors, en M. Hersen R.M. Eisler y P.M. Miller (Eds.), Progress in behavior modification, Newbury Park, California, Sage.

Trujols, J.; Tejero, A.; Casas, M. (1997): Estructura factorial, consistencia interna y eficacia discriminativa del inventario de procesos de cambio para adictos a la heroína. Adicciones, Vol. 9, n³, pag. 331-345.

San Narciso, G.I.; Carreño, J.E.; Pérez, S.F.; Álvarez, C.E.; González, M.P.; Bobes, J. (1998): Evolución de los trastornos de personalidad evaluados mediante el IPDE en una muestra de pacientes heroinómanos en tratamiento con naltrexona. Adicciones, Vol. 10. n.1, pag. 7-21.

\section{AGRADECIMIENTOS}

Este trabajo ha sido realizado gracias a la beca concedida por el Instituto de Investigación en Drogodependencias (INID). Universidad Miguel Hernández. 